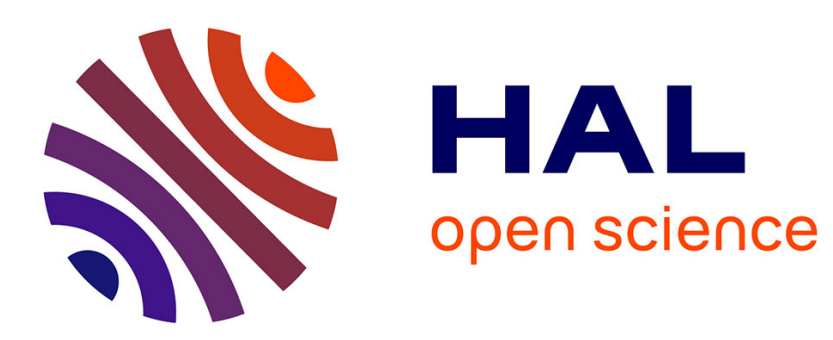

\title{
Finite Element Model of Soil Water and Nutrient Transport with Root Uptake: Explicit Geometry and Unstructured Adaptive Meshing
}

Pierre-Henri Tournier, Frédéric Hecht, Myriam Comte

\section{- To cite this version:}

Pierre-Henri Tournier, Frédéric Hecht, Myriam Comte. Finite Element Model of Soil Water and Nutrient Transport with Root Uptake: Explicit Geometry and Unstructured Adaptive Meshing. Transport in Porous Media, 2015, 106 (2), pp.487-504. 10.1007/s11242-014-0411-7 . hal-01102829

\section{HAL Id: hal-01102829 \\ https: / hal.sorbonne-universite.fr/hal-01102829}

Submitted on 13 Jan 2015

HAL is a multi-disciplinary open access archive for the deposit and dissemination of scientific research documents, whether they are published or not. The documents may come from teaching and research institutions in France or abroad, or from public or private research centers.
L'archive ouverte pluridisciplinaire HAL, est destinée au dépôt et à la diffusion de documents scientifiques de niveau recherche, publiés ou non, émanant des établissements d'enseignement et de recherche français ou étrangers, des laboratoires publics ou privés. 


\title{
Finite element model of soil water and nutrient transport with root uptake: explicit geometry and unstructured adaptive meshing
}

\author{
Pierre-Henri Tournier · Frédéric Hecht · Myriam Comte
}

\begin{abstract}
In this paper, we consider a model of soil water and nutrient transport with plant root uptake. The geometry of the plant root system is explicitly taken into account in the soil model. We first describe our modeling approach. Then, we introduce an adaptive mesh refinement procedure enabling us to accurately capture the geometry of the root system and small-scale phenomena in the rhizosphere. Finally, we present a domain decomposition technique for solving the problems arising from the soil model as well as some numerical results.
\end{abstract}

Keywords finite element method; unstructured mesh adaptation; domain decomposition; plant root uptake

\section{Introduction}

Numerous models have been developed in the past in order to adress the different spatial and temporal scales relevant to soil water and nutrient transport and uptake by plant roots, from crop models used to predict yields at the field level to recent plant based models involving the explicit architectural description of root system development.

Spatially explicit models defining 3D plant architecture are designed to investigate the relationship between root architectural traits and the spatio-temporal variability of resource supply. They are providing insights for understanding various root-soil interactions over a range of spatial scales and aid in the design of agricultural management schemes for improving plant performance in specific environments.

However, simulation of water and nutrient uptake is challenging especially if we consider spatial heterogeneities and local soil conditions in the rhizosphere around the roots, which are often quite different from those in the bulk soil. In addition, the efficiency of yield enhancement techniques depends on responsive root growth which allows plants to forage with precision in an heterogeneous environment.

This work is an attempt to include and resolve accurately local rhizosphere processes occurring at the individual root level in explicit plant scale models by taking advantage of the recent advances of scientific computing in the field of adaptive meshing and parallel computing.

The mechanistic model described here can be used to investigate plant-soil relationships in specific situations through for example sensitivity analysis, as well as verifying hypotheses and simplifications that are made in other models. Such applications will be the focus of subsequent papers.

The model is comparable to [7,11] where a discretization technique based on regular grids is employed. In such models, soil-root fluxes are taken into account in soil voxels by averaging and distributing between the soil nodes. In this work, we develop a new approach that takes advantage of the flexibility of adaptive refinement of unstructured finite element meshes to resolve small-scale behaviours such as the local hydraulic conductivity drop near the soil-root interface while retaining the simplicity of the standard finite element method. Adaptive unstructured volume remeshing is quite a powerful tool when considering complex structures such as plant root systems.

P.-H. Tournier E-mail: tournier@ann.jussieu.fr·F. Hecht E-mail: frederic.hecht@upmc.fr· M. Comte E-mail: comte@ann.jussieu.fr Université Pierre et Marie Curie - Paris 6, UMR 7598 Laboratoire Jacques-Louis Lions, Paris, F-75005 France 
The paper is organized as follows: section 2 describes our water model. We consider that the root system can be represented as a tree-like network composed of cylindrical root segments. We then define radial and axial water flows on this network that can be coupled to the soil model via a sink term in the Richards equation. The sink term is constructed upon a characteristic function representative of the geometry of the root system. In section 3, the nutrient model is presented in a similar way. Section 4 briefly describes the standard finite element method used in this work. In section 5, the adaptive mesh refinement algorithm is presented: the characteristic function of the root system is computed and then used to construct a metric field in order to drive the mesh adaptation procedure. Since such an approach is computationally intensive, a parallelization technique based on a scalable Schwarz domain decomposition method is used to solve the problems arising from the soil and nutrient models. The procedure is highlighted in section 6 .

Throughout this paper the soil domain is denoted by $\Omega \subset \mathbb{R}^{d}(d=2,3)$. We consider the evolution of the water potential and nutrient concentration for $t \in[0, T], T>0$.

\section{The water model}

\subsection{The Richards equation}

In soils, water movement is governed by the Richards equation. Richards equation is derived from the continuity equation

$$
\frac{\partial \theta}{\partial t}+\nabla \cdot q=S
$$

with $\theta$ the volumetric water content, $q$ the macroscopic Darcy flow and $S$ representing sources/sinks.

Darcy law relates the water flow to the pressure of the water at any time $t$ :

$$
q=-K \nabla \mathcal{H},
$$

where $K$ is the hydraulic conductivity and $\mathcal{H}$ is the total hydraulic head (water potential on weight basis), which can be expressed as

$$
\mathcal{H}=h+z .
$$

Here, $h$ is the pressure head and comes from a hydrostatic pressure if $h>0$ and from a capillary pressure if $h<0$. $z$ is the height against the gravitational direction.

The volumetric water content $\theta$ and the hydraulic conductivity $K$ are linked to the pressure head $h$ by relationships that depend on the soil properties.

By combining (2.1) and (2.2) we obtain the Richards equation:

$$
\partial_{t}(\theta(h))-\nabla \cdot(K(h) \nabla(h+z))=S \quad \text { in } \quad[0, T] \times \Omega .
$$

Equation (2.4) is subject to the initial condition

$$
h(x, 0)=h_{0}(x) \text { in } \Omega,
$$

and the no-flux boundary condition

$$
K(h) \nabla(h+z) \cdot \mathbf{n}=0 \quad \text { on } \quad[0, T] \times \partial \Omega,
$$

where $\mathbf{n}$ denotes the unit outward normal to the boundary of the domain $\Omega$.

The $\theta(h)$ and $K(h)$ relationships are given by empirical models whose parameters depend on the soil physical properties. We use the Brooks-Corey model:

$$
\begin{aligned}
& \Theta(h):=\frac{\theta(h)-\theta_{m}}{\theta_{M}-\theta_{m}}=\left[\frac{h}{h_{b}}\right]^{-\lambda}:=\left\{\begin{array}{c}
\left(\frac{h}{h_{b}}\right)^{-\lambda} \text { for } \quad h \leq h_{b} \\
1 \quad \text { for } \quad h \geq h_{b},
\end{array}\right. \\
& K(h)=K_{s}\left[\frac{h}{h_{b}}\right]^{-\lambda e(\lambda)} \quad \text { with } e(\lambda):=3+\frac{2}{\lambda},
\end{aligned}
$$


where $\Theta$ is the normalized water content.

The parameters of the model are defined as follows:

- $\theta_{M}$ is the saturated water content.

- $\theta_{m}$ is the residual water content.

- $K_{s}$ is the saturated hydraulic conductivity.

$-h_{b}$ is the bubbling pressure head.

$-\lambda$ is the pore size distribution index.

Experimental evidence has shown that a cycle of wetting-drying of a soil exhibits hysteresis: the water content has different profiles with respect to the wetting and draining processes. This effect can be of importance when considering irrigation or rainfall together with root water uptake. Although hysteresis effects are neglected in the model described here, hysteresis in the soil water retention function $\theta(h)$ can be taken into account by including empirical hysteresis models such as [16] based on main wetting and drying curves.

We introduce the Kirchhoff transformation $\kappa$ which enables us to reduce the nonlinearity of Richards equation:

$$
\kappa: h \rightarrow p \int_{0}^{h} K(p) d p
$$

The new variable $p$ is called the generalized pressure. Previous applications of the Kirchhoff transformation to Richards equation can be found for example in $[20,25,12,23]$.

The water content as a function of $p$ is denoted by

$$
M(p):=\theta\left(\kappa^{-1}(p)\right)
$$

Using the chain rule, we have

$$
\nabla p=K(h) \nabla(h) .
$$

Thus the Richards equation reads:

$$
\partial_{t}(M(p))-\nabla \cdot\left(\nabla p+K\left(\kappa^{-1}(p)\right) \nabla z\right)-S=0 \quad \text { in } \quad[0, T] \times \Omega .
$$

The transformed equation is a semilinear equation in which the nonlinearity in front of the spatial derivative has been eliminated.

Using the backward Euler scheme for the time discretization, we are able to write the following weak formulation of the semi-discrete problem: find $p^{n+1} \in H^{1}(\Omega)$ such that $\forall v \in H^{1}(\Omega)$,

$$
\int_{\Omega} \frac{M\left(p^{n+1}\right)-M\left(p^{n}\right)}{\Delta t} v+\int_{\Omega} \nabla p^{n+1} \nabla v+\int_{\Omega} K\left(\kappa^{-1}\left(p^{n+1}\right)\right) \nabla z \nabla v-\int_{\Omega} S v=0 .
$$

Following the approach suggested in $[4,23]$, the solution at each time step is obtained iteratively; applying Newton's method to linearize $M\left(p^{n+1}\right)$ gives the following Newton-like iteration where $i$ is the inner iteration counter for time $n+1$ :

$$
\int_{\Omega} \frac{M^{\prime}\left(p^{i}\right)\left(p^{i+1}-p^{i}\right)+M\left(p^{i}\right)-M\left(p^{n}\right)}{\Delta t} v+\int_{\Omega} \nabla p^{i+1} \nabla v+\int_{\Omega} K\left(\kappa^{-1}\left(p^{i}\right)\right) \nabla z \nabla v-\int_{\Omega} S v=0 .
$$

In practice, as the soil dries the capillary effects get stronger as well as the nonlinearities, while the gravity term becomes of less importance. This allows us to use a simple picard method for the gravity term with no effect on the convergence rate.

The use of the Brooks-Corey model allows us to express the Kirchhoff transformation and its inverse and the transformed functions involved in (2.13) explicitly in a closed form as in [3]: 


$$
\begin{aligned}
& p=\kappa(h)=\left\{\begin{aligned}
\frac{h_{b}}{-\lambda e(\lambda)+1}\left(\frac{h}{h_{b}}\right)^{-\lambda e(\lambda)+1}+\frac{-\lambda e(\lambda) h_{b}}{-\lambda e(\lambda)+1} \text { for } & h \leq h_{b} \\
h & \text { for } \quad h \geq h_{b},
\end{aligned}\right. \\
& h=\kappa^{-1}(p)=\left\{\begin{array}{cl}
h_{b}\left(\frac{p(-\lambda e(\lambda)+1)}{h_{b}}+\lambda e(\lambda)\right)^{\frac{1}{-\lambda e(\lambda)+1}} & \text { for } \quad p_{c}<p \leq h_{b} \\
p & \text { for } \quad p \geq h_{b},
\end{array}\right. \\
& M(p)= \begin{cases}\theta_{m}+\left(\theta_{M}-\theta_{m}\right)\left(\frac{p(-\lambda e(\lambda)+1)}{h_{b}}+\lambda e(\lambda)\right)^{\frac{\lambda}{\lambda e(\lambda)-1}} & \text { for } \quad p_{c}<p \leq h_{b} \\
\theta_{M} & \text { for } \quad p \geq h_{b},\end{cases} \\
& M^{\prime}(p)=\left\{\begin{array}{cl}
\left(\theta_{M}-\theta_{m}\right) \frac{-\lambda}{h_{b}}\left(\frac{p(-\lambda e(\lambda)+1)}{h_{b}}+\lambda e(\lambda)\right)^{\frac{\lambda}{\lambda e(\lambda)-1}-1} & \text { for } \quad p_{c}<p \leq h_{b} \\
0 & \text { for } \quad p \geq h_{b},
\end{array}\right. \\
& K\left(\kappa^{-1}(p)\right)=\left\{\begin{array}{cll}
\left(\frac{p(-\lambda e(\lambda)+1)}{h_{b}}+\lambda e(\lambda)\right)^{\frac{\lambda e(\lambda)}{\lambda e(\lambda)-1}} & \text { for } \quad p_{c}<p \leq h_{b} \\
1 & \text { for } \quad p \geq h_{b},
\end{array}\right.
\end{aligned}
$$

Since the transformations (2.14) are obtained analytically, the additional computational cost of employing the Kirchhoff transformation is negligible compared to that of solving the linear system arising from the discretization of (2.13).

Note that the discontinuity of $M^{\prime}(p)$ for $p=h_{b}$ comes from the non-differentiability of the Brooks-Corey function $\theta(h)$ at $h=h_{b}$. However, we do not consider the saturated case $h \geq h_{b}$ in the examples presented in this paper. Besides, from a numerical point of view, numerical tests have shown that for realistic parameter values the discontinuity is small and does not hinder the convergence of the iterative method.

\subsection{Root water uptake}

Here we consider that the root system is composed of cylindrical root segments. It can then be represented as a series of interconnected nodes forming a network of segments $\Sigma$, each segment with its own parameters (radius, conductivity, etc.). Such a representation can be generated by the Matlab code RootBox [18], which implements a root system growth model based on L-Systems. RootBox is a root architectural model that explicitly simulates the architecture of root systems in the 3D space, using a set of growth rules which are applied to a series of root types or classes, with each root type having its own characteristic set of growth parameters such as root elongation rate or branching density. The algorithm computes elongation and branching of the roots according to the initial growth speed, lengths of apical and basal zones as well as internodal distances, maximal number of branches and branching angles. Growth direction can follow different types of user defined tropisms. The model has a stochastic component in that all parameters can be given with mean and standard deviation. Fig. 1 shows an example of a maize root system generated by RootBox.

In the following we define water flows on the root network and describe the coupling with the soil model as was done in [7].

We make the same assumptions as in [7], which are explained in [6]. The model describing root water uptake can be found in $[8,17]$ : water flow in roots and between soil and roots is described by the transpiration-cohesiontension mechanism and follows an Ohm's law analogy.

Let us recall the hypotheses made in [6]: First, the influence of solutes on flow is neglected, because during periods of active transpiration, the hydrostatic pressure gradient rather than the osmotic potential gradient is the effective driving force for flow. The second hypothesis consists in neglecting the capacitive effect of the roots and considering only steady-state flow, because water stored in roots is generally small compared to transpiration requirements. Thus, for a cylindrical root segment of radius $r$ and length $l$ and following [17], we can define the volumetric radial water flow into the root from the soil $J_{r}$ and the longitudinal flow up the root in the xylem $J_{x}$ as

$$
\begin{aligned}
& J_{r}=L_{r} s_{r}\left(h_{s}-h_{r}\right), \\
& J_{x}=-K_{r} \frac{d h_{r}}{d l},
\end{aligned}
$$




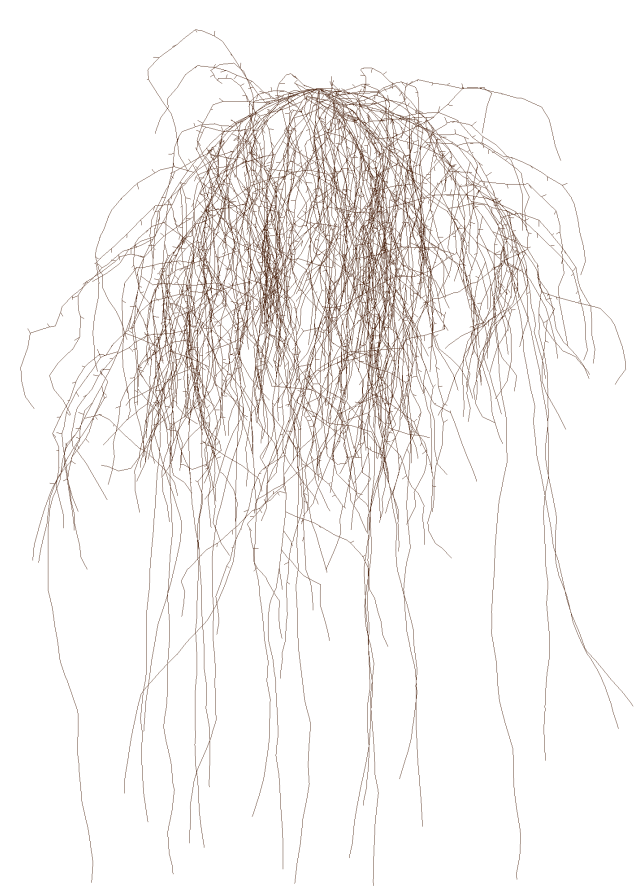

Fig. 1 Example of a 20-days-old maize root system generated by RootBox composed of 10611 segments

where

- $L_{r}$ is the radial conductivity of the root and represents the conductivity of the series of tissues from the root surface to the xylem,

- $K_{r}$ is the xylem conductance,

- $s_{r}=2 \pi r l$ is the root-soil interface area,

- $h_{s}$ is the soil water potential at the root surface,

- $h_{r}$ is the water potential in the xylem.

Although these simplifications are made in our model as well, the model can be extended by taking into account osmotic gradients and capacitive effects of roots.

Equations (2.15) giving radial and longitudinal flows can be used to formulate a water mass balance equation for a given root node $i$ of parent node $p$ in the tree-like structure as depicted in Fig. 2:

$$
J_{x, i}=\sum_{j \in \operatorname{childs}(i)} J_{x, j}+J_{r, i}
$$

which can be written as

$$
-K_{r, i} \frac{h_{r, p}-h_{r, i}}{l_{i}}=-\sum_{j \in \operatorname{childs}(i)}\left(K_{r, j} \frac{h_{r, i}-h_{r, j}}{l_{j}}\right)+L_{r, i} 2 \pi r_{i} l_{i} \frac{\left(h_{s, i}-h_{r, i}\right)+\left(h_{s, p}-h_{r, p}\right)}{2} .
$$

Here $K_{r, i}, L_{r, i}, r_{i}$ and $l_{i}$ refer to the root segment $(p, i)$ while $K_{r, j}$ and $l_{j}$ relate to the root segment $(i, j) . h_{s, i}$ and $h_{r, i}$ are the soil water potential at root node $i$ and the xylem water potential at root node $i$ respectively. We approximate the potentials $h_{s}$ and $h_{r}$ for segment $(i, p)$ by averaging their value at the two nodes $i$ and $p$. Parameters $L_{r}$ and $K_{r}$ are given for each segment and can depend on various data such as root type and age.

Writing (2.17) for every node in the tree-like structure, the xylem water potential vector $\left(h_{r, i}\right)_{i}$ is then solution of a linear system, with the right-hand side containing the soil factors represented by the $h_{s, i}$.

At the root collar, we can prescribe the transpiration flow or the xylem potential with a Neumann or Dirichlet boundary condition respectively. We can follow the same approach as in [7,11]: In the case of a flux-type boundary condition, stress may occur when the evaporative demand cannot be met by the soil. In such a case, a maximum 


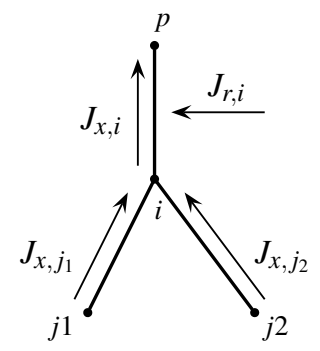

Fig. 2 Water mass balance for root node $i$

allowable threshold value for absolute collar water potential is defined (usually taken as a typical value of the permanent wilting point $h_{w}=-150 \mathrm{~m}$ ), beyond which the collar boundary condition is switched from a flux-type (Neumann) to a pressure-head-type (Dirichlet) condition.

Other models could also be considered, such as [24] where stomatal response to a drying soil is modeled by a logistic function with empirically determined parameters.

In order to take the radial water uptake flows into account in the soil water model, a sink term $S$ in the Richards equation is defined in the domain. Since the sink term represents root uptake flows in the 3D (or 2D) space, we construct $S$ through a characteristic function of the root system $f_{c}$ representative of its geometry using the distance function to the root network $\Sigma$. The characteristic function $f_{c}$ can be seen as a smooth approximation of the 1D root network $\Sigma$, taking the values 1 at the root and 0 away from the root, with a smooth change of width $\varepsilon$ in between.

The purpose of the characteristic function is threefold: define a regularization of the delta function representing the network of segments $\Sigma$, construct a sink term matching the volume occupied by the roots by using the diameter of the root as the width of the regularization, and drive the adaptive mesh refinement procedure.

The function $f_{c}$ representative of the geometry of the root system in the domain is constructed as follows:

- For a point $x$ of the domain $\Omega$ the distance $d$ from $x$ to the root is computed:

$$
d(x)=\min _{s \in \Sigma} d_{s}(x)
$$

with $\Sigma$ the set of root segments in the tree-like network. For each root segment $s$, the distance $d_{s}(x)$ from the point $x$ to the segment $s$ is easily computed using distance from line and point routines.

- The distance function $d$ is then used to compute the characteristic function. There is a variety of admissible transformations that we can use, and we choose the following:

$$
f_{c}(x)=f_{d}(d(x))=1-\tanh \left(\frac{6 d(x)}{\varepsilon}\right) .
$$

We can choose $\varepsilon$ to be equal to the radius of the root.

We can now build the sink term in the Richards equation. In order to ensure that the sink term in the soil model corresponds to the volumetric radial flow in the network model, we need to introduce a scale factor depending on the choice of $f_{d}$.

Let us consider the case of a cylindrical root segment $s$, formed by the nodes $i$ and $j$. The corresponding radial flow is

$$
J_{r}=L_{r} 2 \pi r_{r} l_{r} \frac{\left(h_{s, i}-h_{r, i}\right)+\left(h_{s, j}-h_{r, j}\right)}{2} .
$$

We want the integral of the corresponding sink term $S$ over the domain to be equal to the outflow rate, i.e.

$$
\int_{\Omega} S=-J_{r}
$$


If $f_{c}$ is the characteristic function of the single root segment as defined above, using cylindrical coordinates we get (in the $3 \mathrm{D}$ case)

$$
\int_{\Omega} f_{c} \simeq 2 \pi l_{r} \int_{0}^{R} r f_{d}(r) d r
$$

with $R>>\varepsilon$. The approximation error coming from the truncature in the integral is negligible for usual choices of $f_{d}$.

Let us define $\operatorname{Tr}$ as

$$
T_{r}=\int_{0}^{R} r f_{d}(r) d r
$$

We then define the sink term $S$ as

$$
S=-f_{c} \frac{L_{r} r_{r}}{T_{r}} h_{l},
$$

where $h_{l}$ only depends on the longitudinal coordinate and linearly interpolates $h_{s}-h_{r}$ along the segment. Thus, we have

$$
\int_{\Omega} S=-\int_{\Omega} f_{c} \frac{L_{r} r_{r}}{T_{r}} h_{l}=-L_{r} 2 \pi r_{r} l_{r} \frac{\left(h_{s, i}-h_{r, i}\right)+\left(h_{s, j}-h_{r, j}\right)}{2}=-J_{r} .
$$

The extension to the whole root system is straightforward.

Since the characteristic function does not correspond to an arrangement of perfect cylindrical root segments due to its shape at root tips or in-between root segments, a modified approach consists in adjusting the surface areas of the root segments in the definition of the radial uptake flows in the root network model so that for each root segment, the volumetric uptake flow is equal to the actual contribution of the segment to the global sink term in the soil model. This approach ensures that the amount of water depleted in the soil water model is equal to the transpiration rate in the network model, although the difference is minimal in actual computations.

The coupling between the tree-like model and the soil water model consists in iteratively solving the two problems until convergence. Let $h_{s}^{t_{i}}$ be the soil matric potential distribution at time $t_{i}, h_{s}^{k}$ and $h_{r}^{k}$ the soil and xylem matric potentials at inner iteration $k$ and time $t_{i+1}$. The coupling algorithm reads as follows:

1. $h_{s}^{0}=h_{s}^{t_{i}}$.

2. Solve the linear system of the tree-like model derived from (2.17) with soil factors $h_{s}^{k}$, obtain $h_{r}^{k}$ on the root network.

3. Compute the sink term $S$ as in (2.24) using $h_{s}^{k}$ and $h_{r}^{k}$.

4. Perform an inner iteration of (2.13), obtain $h_{s}$ in the soil domain.

5. $h_{s}^{k+1}=h_{s}^{k}+\alpha_{k}\left(h_{s}-h_{s}^{k}\right)$, where $\alpha_{k}$ is an under-relaxation parameter that ensures convergence of the system.

6. If $\left\|h_{s}-h_{s}^{k}\right\|>\tau$, go to 2 . with $k \leftarrow k+1$.

Fig. 3 gives an overview of the water model through an example.

\section{The nutrient model}

Here we consider the evolution of the concentration $c$ of a nutrient ion $N$ in the soil solution, governed by diffusion, mass flow, adsorption in the soil solid phase and root uptake.

\subsection{The convection-diffusion equation}

The convection-diffusion equation expresses the nutrient mass balance and can be written in its conservative form:

$$
\partial_{t}(\theta c+\varphi(c))=\nabla \cdot(A \nabla c-q c)+S_{c}(c) \quad \text { in } \quad[0, T] \times \Omega,
$$

where 


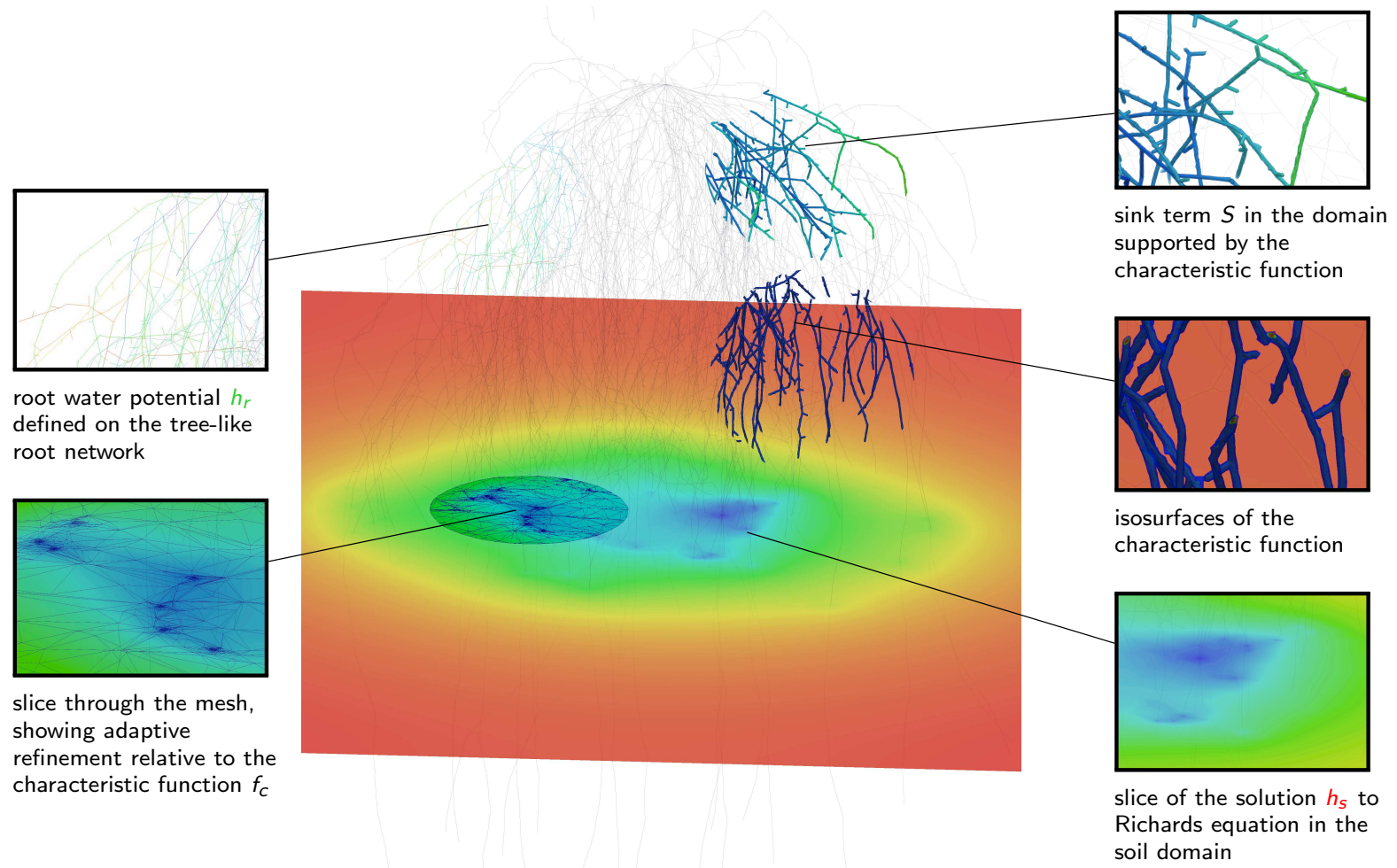

Fig. 3 Overview of the water model

- $A$ is the diffusion coefficient of $N$ in the soil. For simplicity we consider a modified diffusion coefficient with a tortuosity factor in place of the effective dispersion coefficient tensor that is usually employed for flow in porous media, although the model can easily be extended to account for dispersibility since water flow is explicitly considered. Following [2], $A$ is given by

$$
A=A_{0} \theta f_{l},
$$

with $A_{0}$ the diffusion coefficient of $N$ in free water and $f_{l}$ the so-called tortuosity factor:

$$
f_{l}=\left\{\begin{array}{cc}
f_{1} \theta+f_{2} \text { for } & \theta \geq \theta_{l}, \\
\frac{\theta\left(f_{1} \theta_{l}+f_{2}\right)}{\theta_{l}} \text { for } & \theta<\theta_{l},
\end{array}\right.
$$

where $f_{1}, f_{2}$ and $\theta_{l}$ are parameters depending on soil properties.

- $\varphi$ is an adsorption/desorption isotherm relating the amount of adsorbed $N$ to the equilibrium concentration of $N$ in solution; we use the Freundlich adsorption isotherm [19], defined by

$$
\varphi(c)=\kappa c^{b},
$$

where $\kappa>0$ and $b \in(0,1)$ are fitting parameters of the model,

- $S_{c}(c)$ represents sources/sinks.

Equation (3.1) is subject to the initial condition

$$
c(x, 0)=c_{0}(x) \quad \text { in } \Omega,
$$

and the no-flux boundary condition

$$
(A \nabla c-q c) \cdot \mathbf{n}=0 \quad \text { on } \quad[0, T] \times \partial \Omega .
$$


The convective form of equation (3.1) is obtained by multiplying equation (2.1) by $c$ and substracting it from equation (3.1). This gives

$$
\left(\theta+\varphi^{\prime}(c)\right) \partial_{t} c=\nabla \cdot(A \nabla c)-q \cdot \nabla c-S c+S_{c}(c) .
$$

Here $\theta+\varphi^{\prime}(c)$ is the buffer power and represents the ability of the soil to resupply nutrients as plants take it up from the soil solution.

The Lagrangian form is derived by dividing by $\theta+\varphi^{\prime}(c)$ and defining the material derivative

$$
\frac{D c}{D t}=\partial_{t} c+\frac{q}{\theta+\varphi^{\prime}(c)} \cdot \nabla c .
$$

We can use the method of characteristics to handle the convective part. The velocity field is $a=\frac{q}{\theta+\varphi^{\prime}(c)}$.

Following [21], we can use the approach yielding (3.7) at the semi-discrete level to reduce mass balance errors. Using the implicit Euler scheme for the time discretization of (3.1) gives

$$
\frac{\theta^{n+1} c^{n+1}+\varphi\left(c^{n+1}\right)-\theta^{n} c^{n}-\varphi\left(c^{n}\right)}{\Delta t}=\nabla \cdot\left(A^{n+1} \nabla c^{n+1}-q^{n+1} c^{n+1}\right)+S_{c}\left(c^{n+1}\right) .
$$

Our implicit time discretization of Richards equation (2.12) corresponds to

$$
\frac{\theta^{n+1}-\theta^{n}}{\Delta t}+\nabla \cdot q^{n+1}=S^{n+1} \text {. }
$$

Multiplying (3.10) by $c^{n+1}$ and substracting it from (3.9), we are able to write the weak formulation of the semidiscrete problem: find $c^{n+1} \in H^{1}(\Omega)$ such that $\forall v \in H^{1}(\Omega)$,

$$
\int_{\Omega} \frac{\theta^{n} c^{n+1}+\varphi\left(c^{n+1}\right)-\left(\theta^{n} c^{n}\right) \circ X-\varphi\left(c^{n} \circ X\right)}{\Delta t} v+\int_{\Omega} A^{n+1} \nabla c^{n+1} \nabla v+\int_{\Omega} S^{n+1} c^{n+1} v-\int_{\Omega} S_{c}\left(c^{n+1}\right) v=0
$$

with the approximated characteristics $X=x-\Delta t a$.

Applying Newton's method and denoting by $i$ the inner iteration counter for time $n+1$, we get

$$
\begin{aligned}
& \int_{\Omega} \frac{\theta^{n} c^{i+1}+\varphi\left(c^{i}\right)+\varphi^{\prime}\left(c^{i}\right)\left(c^{i+1}-c^{i}\right)-\left(\theta^{n} c^{n}\right) \circ X-\varphi\left(c^{n} \circ X\right)}{\Delta t} v+\int_{\Omega} A^{n+1} \nabla c^{i+1} \nabla v \\
& +\int_{\Omega} S^{n+1} c^{i+1} v-\int_{\Omega} S_{c}\left(c^{i}\right) v-\int_{\Omega} S_{c}^{\prime}\left(c^{i}\right)\left(c^{i+1}-c^{i}\right) v=0 .
\end{aligned}
$$

\subsection{Nutrient uptake}

The root acts as a selective membrane for ion uptake. Nutrient uptake by roots is given by a model of enzyme kinetics, relating in this case the root uptake rate of $\mathrm{N}$ to its concentration at the root surface. Here we use the following Michaelis-Menten model:

$$
h(c)=\frac{F_{m} c}{K_{m}+c},
$$

where $h(c)$ is the uptake rate and $F_{m}, K_{m}>0$ are parameters of the model.

This model of active nutrient uptake is taken from [1]. The hypothesis that active uptake becomes more important under low nutrient supply, while the transpiration driven mass flow dominates for higher concentrations, is proposed in [27].

For a cylindrical root segment of radius $r$ and length $l$ and assuming that (3.13) we can define the volumetric nutrient uptake rate by

$$
J_{N}=2 \pi r l h\left(c_{s}\right),
$$

where $c_{s}$ is the concentration of $\mathrm{N}$ at the root surface.

Similarly to the sink term (2.24) in the Richards equation, the sink term $S_{c}$ representing nutrient uptake in the convection-diffusion equation is constructed as follows:

$$
S_{c}=-f_{c} \frac{r}{T_{r}} h(c) .
$$


The model can easily be adapted to implement other nutrient uptake models. For example, in $[22,10]$ the solute uptake term is defined as

$$
S^{\prime}(c)=\varepsilon S c+(1-\varepsilon) S_{c}(c),
$$

where $\varepsilon \in[0,1]$ is a coefficient partitioning total uptake between passive uptake $S c$ where solute enters the root dissolved in water and active uptake $S_{c}(c)$, which could also be described following for example [15] by a MichaelisMenten-type kinetic with a linear component.

\section{Finite element formulation}

In this section we describe briefly the Galerkin P1 finite element approximation of problems (2.13) and (3.12). Let $T_{h}$ be a mesh of the domain $\Omega$. Let

$$
V_{h}=\left\{u_{h} \in H^{1}(\Omega)\left|u_{h}\right|_{K} \in P_{1}, \forall K \in T_{h}\right\} .
$$

Spatial discretization of Equation (2.13) leads to the following discrete variational problem: find $p_{h} \in V_{h}$ such that $\forall v_{h} \in V_{h}$,

$$
\begin{aligned}
\left(M^{\prime}\left(p_{h}^{i}\right) p_{h}, v_{h}\right)+\Delta t\left(\nabla p_{h}, \nabla v_{h}\right)= & \left(M^{\prime}\left(p_{h}^{i}\right) p_{h}^{i}, v_{h}\right)-\left(M\left(p_{h}^{i}\right), v_{h}\right)+\left(M\left(p_{h}^{n}\right), v_{h}\right) \\
& -\left(K\left(\kappa^{-1}\left(p_{h}^{i}\right)\right) \nabla z, \nabla v_{h}\right)+\left(S_{h}^{i}, v_{h}\right) .
\end{aligned}
$$

Spatial discretization of Equation (3.12) leads to the following discrete variational problem: find $c_{h} \in V_{h}$ such that $\forall v_{h} \in V_{h}$,

$$
\begin{aligned}
& \left(\theta_{h}^{n} c_{h}, v_{h}\right)+\left(\varphi^{\prime}\left(c_{h}^{i}\right) c_{h}, v_{h}\right)+\left(A_{h}^{n+1} \nabla c_{h}, \nabla v_{h}\right)+\Delta t\left(S_{h}^{n+1} c_{h}, v_{h}\right)-\Delta t\left(S_{c}^{\prime}\left(c^{i}\right)_{h} c_{h}, v_{h}\right) \\
& =-\left(\varphi\left(c_{h}^{i}\right), v_{h}\right)+\left(\varphi^{\prime}\left(c_{h}^{i}\right) c_{h}^{i}, v_{h}\right)+\left(\left(\theta_{h}^{n} c_{h}^{n}\right) \circ X, v_{h}\right)+\left(\varphi\left(c_{h}^{n} \circ X\right), v_{h}\right)+\Delta t\left(S_{c}\left(c^{i}\right)_{h}, v_{h}\right)-\Delta t\left(S_{c}^{\prime}\left(c^{i}\right)_{h} c_{h}^{i}, v_{h}\right) .
\end{aligned}
$$

Numerical resolution of systems (4.2) and (4.3) is carried out using the finite element software FreeFem++ [9].

\section{Mesh adaptation}

The soil domain $\Omega$ is first represented by a regular initial simplicial finite element mesh. Since the characteristic function of the root system $f_{c}$ is poorly represented on the initial mesh, we refine it iteratively using anisotropic mesh adaptation. Since we expect high gradients and small-scale phenomena to be localized near the roots (i.e. where $f_{c}$ exhibits strong variations), this type of a priori refinement is adequate.

The main steps of the adaptive procedure are as follows: First, we compute $f_{c}$ for each node of the mesh. Then we define a nodal based anisotropic metric from the Hessian of the function $f_{c}$. Finally, the mesh is adapted using the size and streching of elements provided by the metric. This procedure is repeated iteratively.

In 2D, we use the built-in adaptive remesher of FreeFem++. For 3D simulations, FreeFem++ is interfaced with mshmet for computing the Hessian-based anisotropic metric and with the anisotropic fully tetrahedral automatic remesher Mmg3d [5] which uses anisotropic Delaunay kernel and local mesh modifications based on a combination of edge flips, edge collapsing, node relocation and vertex insertion operations to adapt the mesh.

Fig. 4 illustrates the mesh adaptation process in a 2D simulation.

\section{Domain decomposition}

Sinces meshes generated as described in section 5 require a considerable number of nodes to be able to adequately resolve the geometry of complex root systems, linear systems resulting from the discrete problems (4.2) and (4.3) can be quite large. In order to reduce computation time, we opted for a parallel divide-and-conquer technique with an additive Schwartz overlapping domain decomposition method.

The initial computational domain is partitioned by metis [14] into a number of subdomains, on which local variational problems are defined. A two-level coarse grid preconditioner taken from [13] is also used to improve the convergence of the domain decomposition method. 


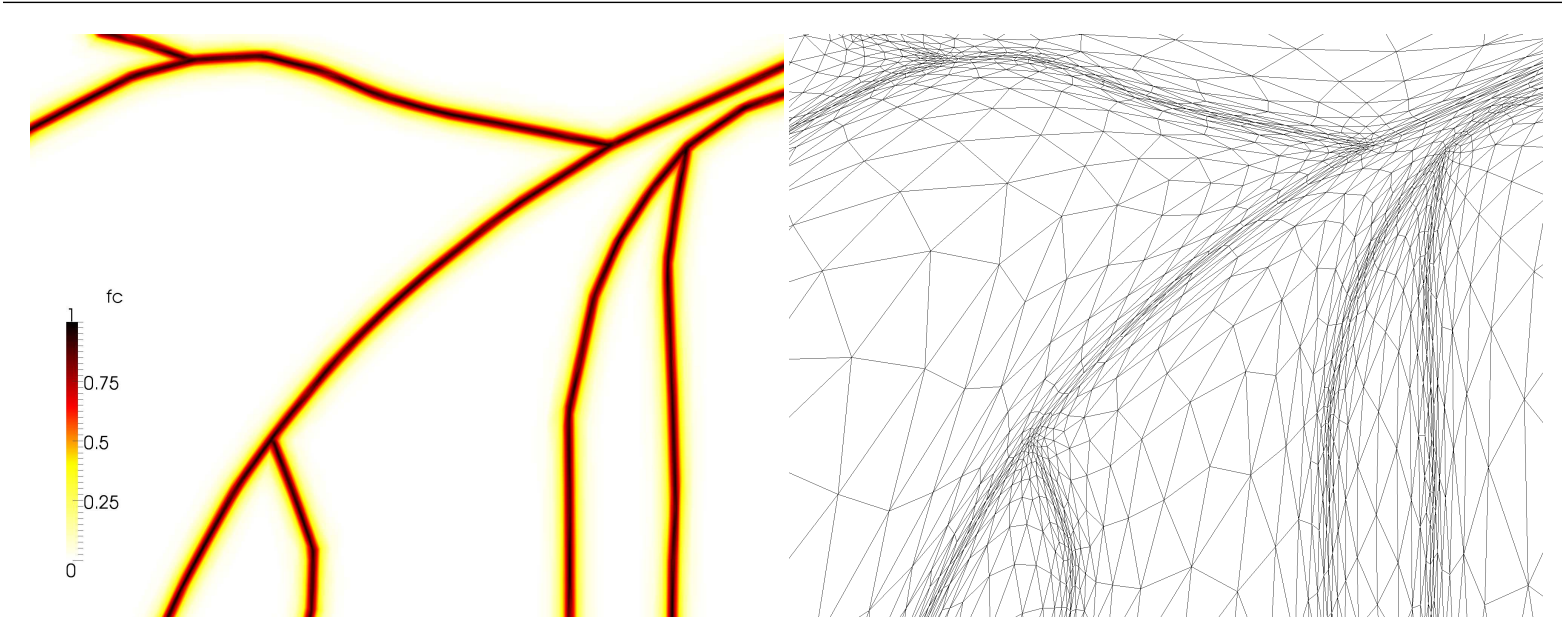

Fig. $42 \mathrm{D}$ example of the mesh adaptation process: representation of the function $f_{c}$ (left) defined on the adapted mesh (right).

Numerical tests are conducted in 2D and in 3D in order to assess the efficiency of the two-level preconditioner compared to a classical one-level preconditioner. We consider one inner iteration of (4.2). In the $2 \mathrm{D}$ case, the mesh is composed of 204331 vertices and 407839 triangles. The 3D mesh is composed of 2673103 vertices and 15273475 tetrahedra.

\begin{tabular}{|c|c|c|c|c|}
\cline { 2 - 5 } \multicolumn{1}{c|}{$2 \mathrm{D}$} & \multicolumn{2}{c|}{ 1-level precond. } & \multicolumn{2}{c|}{ 2-level precond. } \\
\hline \# of subdomains & \# of iterations & Wall-clock time & \# of iterations & Wall-clock time \\
\hline 16 & 42 & $20.95 \mathrm{~s}$ & 11 & $6.69 \mathrm{~s}$ \\
64 & 55 & $4.88 \mathrm{~s}$ & 14 & $1.58 \mathrm{~s}$ \\
140 & 68 & $2.08 \mathrm{~s}$ & 16 & $0.69 \mathrm{~s}$ \\
\hline
\end{tabular}

\begin{tabular}{|c|c|c|c|c|}
\cline { 2 - 5 } \multicolumn{1}{c|}{$3 \mathrm{D}$} & \multicolumn{2}{c|}{ 1-level precond. } & \multicolumn{2}{c|}{ 2-level precond. } \\
\hline \# of subdomains & \# of iterations & Wall-clock time & \# of iterations & Wall-clock time \\
\hline 16 & 31 & $209.93 \mathrm{~s}$ & 17 & $129.15 \mathrm{~s}$ \\
64 & 39 & $29.24 \mathrm{~s}$ & 15 & $13.17 \mathrm{~s}$ \\
140 & 44 & $12.49 \mathrm{~s}$ & 16 & $5.54 \mathrm{~s}$ \\
\hline
\end{tabular}

There are several iterative algorithms used to obtain the numerical solution. In the water model, the outer loop consists in a fixed point algorithm solving alternatively the root problem (2.18) and the soil problem (4.2). Solving the linear system resulting from the discrete soil problem (4.2) using the domain decomposition method presented in this section constitutes an inner loop. Local problems defined on each subdomain are also solved iteratively using a conjugate gradient method, and thus the complete algorithm consists in three nested loops.

We can take advantage of the iterative nature of the domain decomposition and linear solvers by using adaptive stopping criteria in order to further reduce the computational time. Here we use simple heuristics expressing that there is no need to continue with iterations in the inner loop once the error from the outer loop starts to dominate. More elaborate stopping criteria can be used, see for example [26] where adaptive stopping criteria based on a posteriori error estimates are derived.

\section{Numerical resolution}

The purpose of the numerical examples presented in this section is to illustrate the capabilities of the numerical model.

Numerical values used in the examples are as follows:

- parameters for a clay soil are $\theta_{m}=0.068, \theta_{M}=0.38, \lambda=0.17, h_{b}=-0.4 \mathrm{~m}, K_{s}=0.144 \mathrm{~m} \mathrm{~d}^{-1}$. 
- the initial water potential in the soil domain is in hydrostatic equilibrium: $h_{0}=-15 \mathrm{~m}-z$.

For simplicity, all root parameters are taken constant across the whole root system. Numerical values of $L_{r}$ and $K_{r}$ for maize are taken from [6]:

- Root radius is set to $5.0 \times 10^{-4} \mathrm{~m}$.

- $L_{r}=1.92308 \times 10^{-4} \mathrm{~d}^{-1}, K_{r}=4.32 \times 10^{-8} \mathrm{~m}^{3} \mathrm{~d}^{-1}$.

As an illustration of the nutrient model, we consider the transport and uptake of nitrate. Parameters for equation (3.2) are taken from [2], Michaelis-Menten constants for maize are taken from [1]:

- $A_{0}=1.6416 \times 10^{-4} \mathrm{~m}^{2} \mathrm{~d}^{-1}, f_{1}=1.58, f_{2}=-0.17, \theta_{l}=0.12$.

$-F_{m}=8.64 \times 10^{-3} \mathrm{~mol} \mathrm{~m}^{-2} \mathrm{~d}^{-1}, K_{m}=2.5 \times 10^{-2} \mathrm{~mol} \mathrm{~m}^{-3}$.

- the homogeneous initial concentration of nitrate in the soil solution is $c_{0}=5 \mathrm{~mol} \mathrm{~m}^{-3}$.

We consider that adsorption of nitrate in the soil solid phase is negligible: $\varphi=0$.

The first numerical simulation involves the 20-days-old maize root system generated by the Matlab code RootBox depicted in Fig. 1. The soil domain is of dimensions $0.4 \mathrm{~m} \times 0.4 \mathrm{~m} \times 0.4 \mathrm{~m}$. No-flux boundary conditions are imposed on the boundaries of the soil domain. A constant transpiration rate equal to $1.44 \times 10^{-4} \mathrm{~m}^{3} \mathrm{~d}^{-1}$ is imposed at the root collar. The time step $\Delta t$ is taken constant equal to 0.05 d. Numerical results are depicted in Figs. 5 and 6. Fig. 5 shows the Darcy flux $q$ in the vicinity of the roots. In the beginning of the simulation, root water uptake is still relatively evenly distributed over the dense upper portion of the root system (left picture, $t=4.8 \mathrm{~h}$ ). As time passes, the uptake pattern is modified. The soil dries in the dense root zone and the root system takes up water from wetter zones (mostly in the deeper part of the soil profile) in order to maintain a constant transpiration rate (right picture, $t=7.3 \mathrm{~d}$ ).

Fig. 6 shows high gradients developing in the vicinity of the roots as the soil dries.

The second example illustrates how we are able to integrate root growth and chemotropism in 2D by coupling the model with the implementation of growth and tropisms in RootBox.

RootBox can simulate root tip response to mechanical soil heterogeneities as well as various types of tropisms like gravitropism, hydrotropism or chemotropism. The specific growth behaviour can be chosen for every root type.

In RootBox, the implementation of tropisms consists in computing the new growth direction by random minimization of an objective function: for each active root tip, several rotations are randomly computed and the one that leads to minimizing the objective function is chosen. Different types of tropisms are realized by choosing appropriate objective functions depending on soil properties (water content, nutrient concentration). In this example, a combination of gravitropism and chemotropism is achieved by defining the objective function as $-\lambda c+z$ where $\lambda>0$ represents the relative strength of chemotropism.

At each time step, an iteration of the following coupling algorithm is performed:

1. RootBox finds the best growth direction for each active root tip through multiple evaluations of the objective function depending on the spatial concentration $c$. RootBox is interfaced with the FreeFem++ finite element code so that the values of the concentration can be determined by interpolation on the mesh. Then, a new tree-like network with new root segments is obtained.

2. A mesh adapted to the new characteristic function $f_{c}$ is obtained from the previous mesh by the mesh adaptation procedure described in section 5.

3. Finite element functions (namely the current soil water potential and $\mathrm{N}$ concentration) are interpolated from the previous mesh to the new mesh.

4. Solve (2.12) and (3.11) and obtain the new soil water potential and $\mathrm{N}$ concentration distributions.

Fig. 7 depicts some results of such a simulation with the initial nitrate concentration set to a linear profile varying from $0 \mathrm{~mol} \mathrm{~m}^{-3}$ at the top left corner of the domain to $10 \mathrm{~mol} \mathrm{~m}^{-3}$ at the bottom right corner. Notice the accumulation of nitrate around some of the roots in the bottom right: as the soil dries out, radial soil-root water flow increases in the bottom right where the soil is wetter, resulting in the mass flow of nitrate bringing more than the root can take up. 

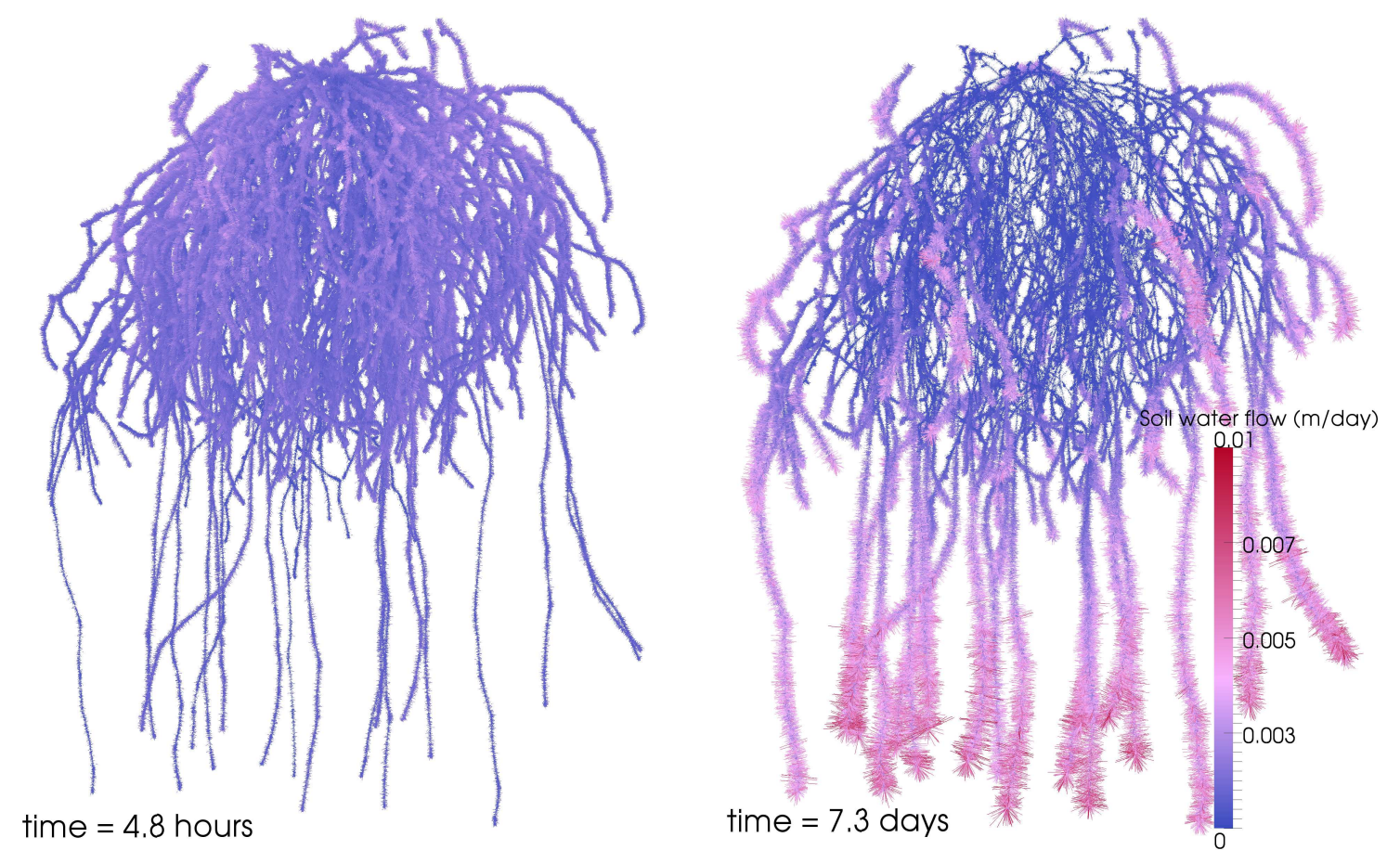

Fig. 5 Soil water flow near the root system of a 20-days-old maize plant

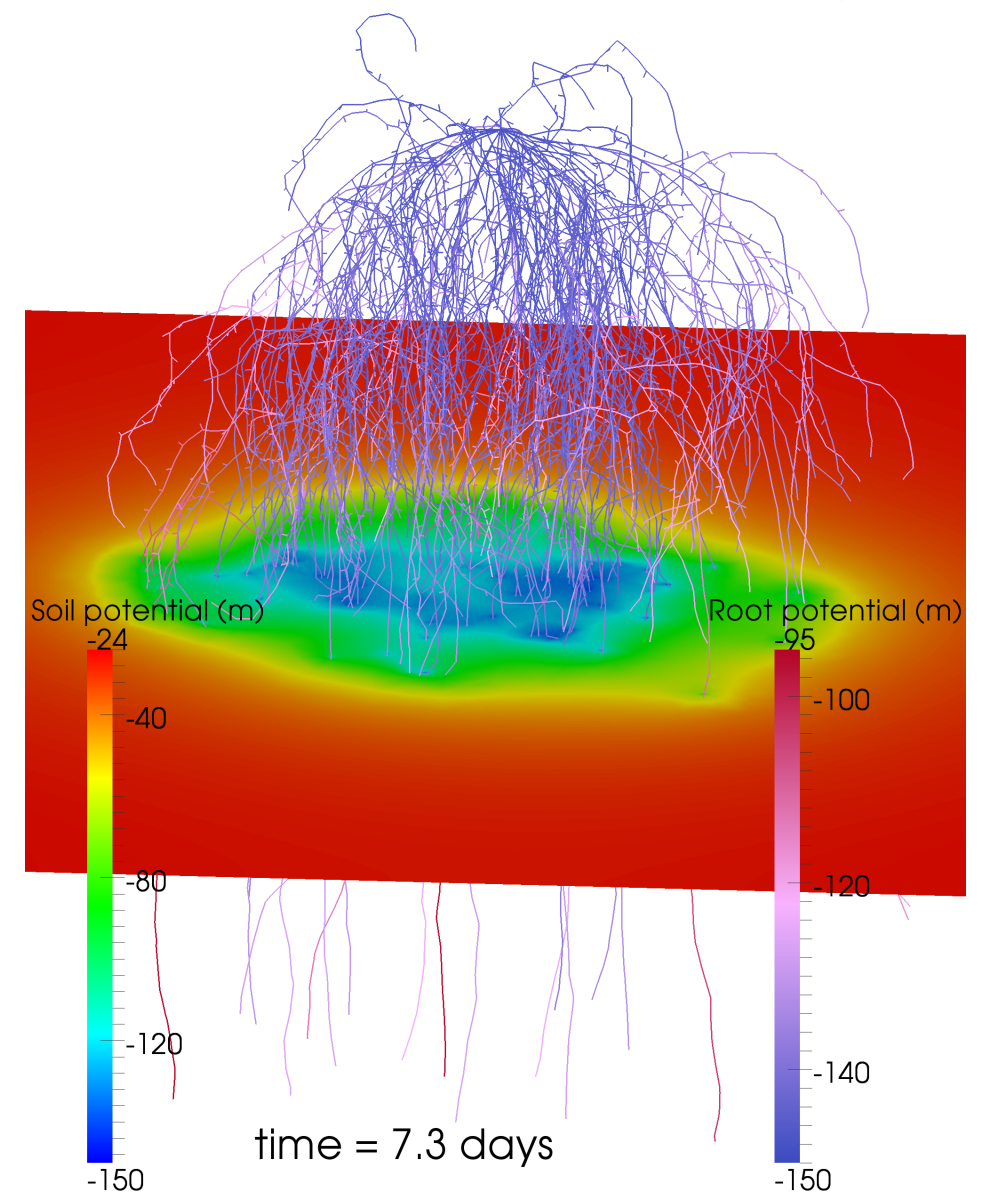

Fig. 6 Root water potential $h_{r}$ defined on the tree-like network and slice of the soil water potential $h_{s}$ 

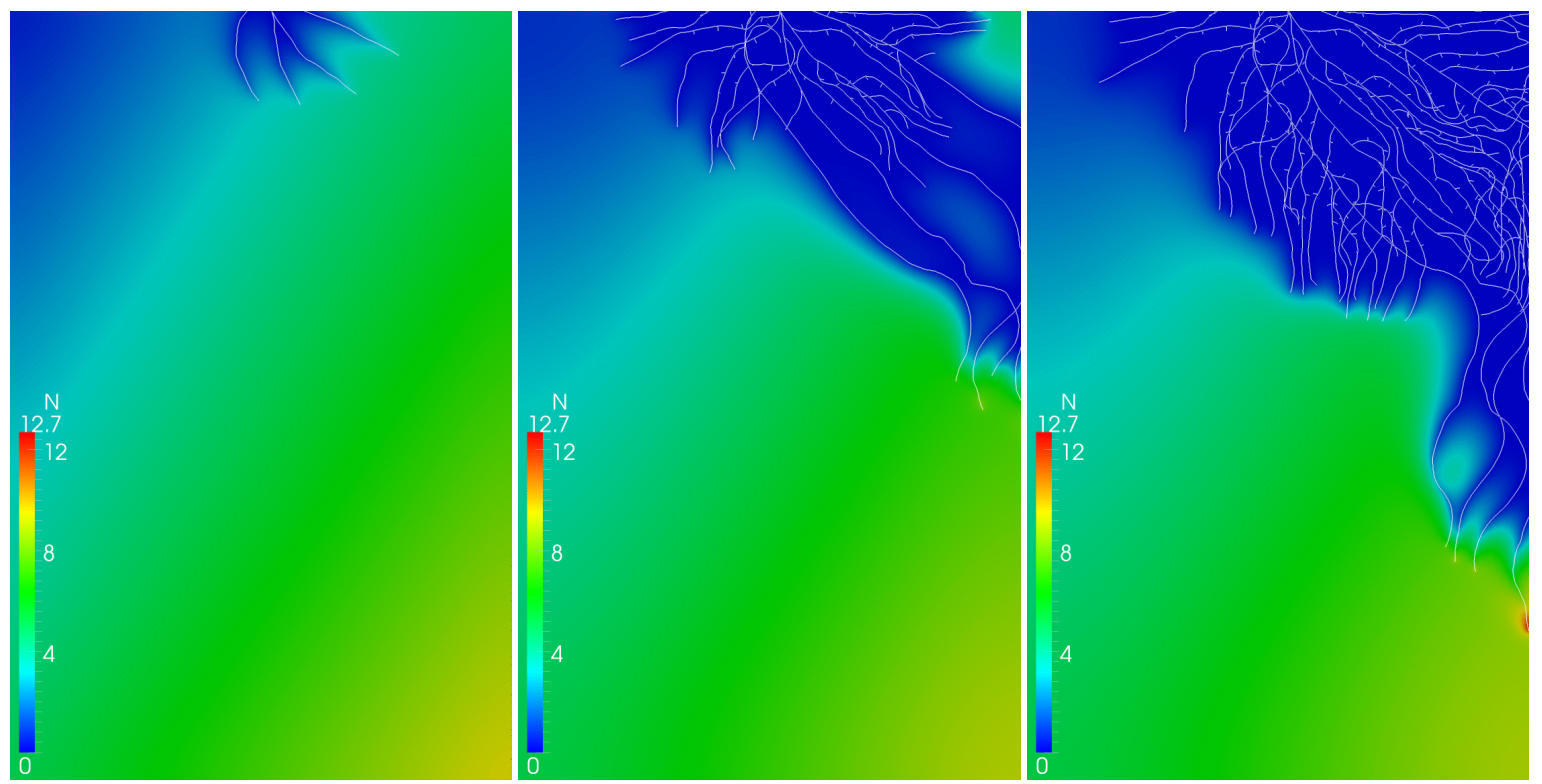

Fig. $72 \mathrm{D}$ simulation example with root growth and chemotropism: snapshots of nitrate concentration at different time steps. In white, isosurface 0.5 of the characteristic function $f_{c}$.

\section{Conclusion}

In this paper we presented a model of soil water and nutrient transport with plant root uptake. A characteristic function of the geometry of the root system was used to construct accurate sink terms corresponding to water and nutrient uptake by roots. An emphasis was put on the spatial discretization with an adaptive mesh refinement procedure producing meshes that are able to resolve the complex geometry of the root system together with smallscale phenomena occuring in the rhizosphere. A parallel finite element method was then presented using a twolevel Schwarz domain decomposition method to solve the potentially large systems arising from the discretization. Numerical experiments were conducted in two and three spatial dimensions to illustrate the capabilities of the model.

Future work will consist in using the diffuse domain approach to approximate the actual surface of the roots in the volume mesh without relying on a surface mesh. The level set representing the root surface splits the domain $\Omega$ into two subdomains $\Omega_{s}$ and $\Omega_{r}$. Following a monolithic approach, different problems are defined on the same mesh in the soil domain $\Omega_{s}$ and in the root system $\Omega_{r}$ using a phase field variable. This approach allows us to make use of the same adaptive volume remeshing procedure that was described in this paper.

Acknowledgements We thank Pierre Jolivet for his valuable insights on FreeFem++ and domain decomposition methods.

\section{References}

1. Barber, S.A.: Soil nutrient bioavailability: a mechanistic approach. Wiley-Interscience, New York (1984)

2. Barraclough, P.B., Tinker, P.B.: The determination of ionic diffusion coefficients in field soils. I. diffusion coefficients in sieved soils in relation to water content and bulk density. Journal of Soil Science 32(2), 225-236 (1981)

3. Berninger, H.: Domain decomposition methods for elliptic problems with jumping nonlinearities and application to the richards equation. Ph.D. thesis, Freie Universität Berlin (2007)

4. Celia, M.A., Bouloutas, E.T., Zarba, R.L.: A general mass-conservative numerical solution for the unsaturated flow equation. Water Resources Research 26(7), 1483-1496 (1990)

5. Dobrzynski, C.: MMG3D: User Guide. Rapport Technique RT-0422, INRIA (2012)

6. Doussan, C., Pagès, L., Vercambre, G.: Modelling of the hydraulic architecture of root systems: An integrated approach to water absorption - model description. Annals of Botany 81, 213-223 (1998) 
7. Doussan, C., Pierret, A., Garrigues, E., Pagès, L.: Water uptake by plant roots: II - modelling of water transfer in the soil root-system with explicit account of flow within the root system - comparison with experiments. Plant and Soil 283(1-2), 99-117 (2006)

8. Fiscus, E.L.: The interaction between osmotic- and pressure-induced water flow in plant roots. Plant Physiology 55(5), 917-922 (1975)

9. Hecht, F.: New development in freefem++. Journal of Numerical Mathematics 20, 251-266 (2013)

10. Hopmans, J.W., Bristow, K.L.: Current capabilities and future needs of root water and nutrient uptake modeling. In: D.L. Sparks (ed.) Advances in Agronomy, Advances in Agronomy, vol. 77, pp. 103 - 183. Academic Press (2002)

11. Javaux, M., Schröder, T., Vanderborght, J., Vereecken, H.: Use of a three-dimensional detailed modeling approach for predicting root water uptake. Vadose Zone Journal 7, 1079-1088 (2008)

12. Ji, S.H., Park, Y.J., Sudicky, E.A., Sykes, J.F.: A generalized transformation approach for simulating steady-state variably-saturated subsurface flow. Advances in Water Resources 31(2), 313-323 (2008)

13. Jolivet, P., Dolean, V., Hecht, F., Nataf, F., Prud'homme, C., Spillane, N.: High performance domain decomposition methods on massively parallel architectures with freefem++. Journal of Numerical Mathematics 20, 287-302 (2013)

14. Karypis, G., Kumar, V.: A fast and high quality multilevel scheme for partitioning irregular graphs. SIAM Journal on Scientific Computing 20, 359-392 (1998)

15. Kochian, L.V., Lucas, W.J.: Potassium transport in corn roots: I. resolution of kinetics into a saturable and linear component. Plant Physiology 70(6), 1723-1731 (1982)

16. Kool, J.B., Parker, J.C.: Development and evaluation of closed-form expressions for hysteretic soil hydraulic properties. Water Resources Research 23(1), 105-114 (1987)

17. Landsberg, J., Fowkes, N.: Water movement through plant roots. Annals of Botany 42(1), 493-508 (1978)

18. Leitner, D., Klepsch, S., Bodner, G., Schnepf, A.: A dynamic root system growth model based on 1-systems. Plant and Soil 332(1-2), 177-192 (2010)

19. McGechan, M., Lewis, D.: Sorption of phosphorus by soil, part 1: Principles, equations and models. Biosystems Engineering 82, 1-24 (2002)

20. Pop, I.S.: Error estimates for a time discretization method for the richards' equation. Computational Geosciences 6(2), 141-160 (2002)

21. Saaltink, M.W., Carrera, J., Olivella, S.: Mass balance errors when solving the convective form of the transport equation in transient flow problems. Water Resources Research 40(5) (2004)

22. Somma, F., Hopmans, J., Clausnitzer, V.: Transient three-dimensional modeling of soil water and solute transport with simultaneous root growth, root water and nutrient uptake. Plant and Soil 202(2), 281-293 (1998)

23. Stevens, D., Power, H.: A scalable and implicit meshless RBF method for the 3D unsteady nonlinear richards equation with single and multi-zone domains. International Journal for Numerical Methods in Engineering 85(2), 135-163 (2011)

24. Tuzet, A., Perrier, A., Leuning, R.: A coupled model of stomatal conductance, photosynthesis and transpiration. Plant, Cell \& Environment 26(7), 1097-1116 (2003)

25. Varado, N., Braud, I., Ross, P., Haverkamp, R.: Assessment of an efficient numerical solution of the 1d richards' equation on bare soil. Journal of Hydrology 323(1-4), 244-257 (2006)

26. Vohralík, M., Wheeler, M.: A posteriori error estimates, stopping criteria, and adaptivity for two-phase flows. Computational Geosciences 17(5), 789-812 (2013)

27. Šimůnek, J., Hopmans, J.W.: Modeling compensated root water and nutrient uptake. Ecological Modelling 220(4), 505-521 (2009) 